\title{
Parameterizing the modified water cloud model to improve soil moisture data retrieval using vegetation models
}

\author{
Kishan Singh RAWAT ${ }^{1}$, Sudhir Kumar SINGH ${ }^{2}$, Ram L. RaY ${ }^{3}$, SzILÁrd SZABÓ ${ }^{4}$ and \\ SANJEEV KUMAR ${ }^{1}$
}

\begin{abstract}
The objective was to parameterize a modified water cloud model using crop coefficients (A and B). These crop coefficients were derived from Landsat- 8 and Sentinel-2 data. Whereas coefficients $C$ and D are of soil parameters. The water cloud model was modified using crop coefficients by minimizing the RMSE between observed VV $\sigma^{0}$ and Sentinel-1 based simulated $\mathrm{VV \sigma} \sigma^{0}$. The comparison with observed and simulated VV polarized $\sigma^{0}$ showed low RMSE $(0.81 \mathrm{~dB})$ and strong $\mathrm{R}^{2}$ of 0.98 for NDVI-EVI combination. However, based on other possible combinations of vegetation indices $\mathrm{VV \sigma}^{0}$ and simulated $\mathrm{VV \sigma} \sigma^{0}$ do not show a good statistical agreement. It was observed that the errors in crop coefficients (A and B) are sensitive to errors in initial vegetation/canopy descriptor parameters.
\end{abstract}

Keywords: NDVI, EVI, SAR, Sentinel, WCM

\section{Introduction}

Soil moisture is an important and key factor that influences the meteorological parameters directly or indirectly; therefore it is important to understand its patterns and cause of variations at the region level (HoRNBERGER, G.M. 1998). The dynamics of soil moisture plays a critical role in the analysis of agricultural drought, weather forecast, flood forecasting, crop yield prediction and climatology (Berthet, L. et al. 2009; BeCK, H.E. et al. 2009; Hegedús, P. et al. 2013, 2015; Dezső, J. et al. 2019). The sensitivity of microwave signals is directly reciprocal to soil dielectric constant, which reflect the soil moisture; microwave signal can penetrate vegetation canopy and provide soil moisture states
(BINDlish, R. et al. 2006). Soil moisture at a regional scale can be observed by the synthetic aperture radar (SAR) with fine spatial and temporal resolutions (SHI, J.C. et al. 1997; Pathe, C. et al. 2009; Paloscia, S. et al. 2013; Pasolli, L. et al. 2015).

Nowadays, many models are available for the quantification of soil moisture at a regional scale, but model complexity and exhaustive data input requirement limit their applications. However, the water cloud model (WCM) requires a lower number of input data (Аттемa, E. and Ulaby, F.T. 1978). The WCM has four empirical coefficients, namely canopy descriptor parameters (A and $\mathrm{B}$ ) and soil parameters (C and D). At local scale to regional analysis, the vegetation/crop coefficients of the WCM

\footnotetext{
${ }^{1}$ Civil Engineering Department, Graphic Era (Deemed to be University), Dehradun - 248002 Uttrakhand, India. E-mails: ksr.kishan@gmail.com, er.sanjeevkr@gmail.com

${ }^{2}$ K. Banerjee Centre of Atmospheric \& Ocean Studies, IIDS, Nehru Science Centre, University of Allahabad, Prayagraj - 21 1002, Uttar Pradesh, India. Corresponding author's e-mail: sudhirinjnu@gmail.com

${ }^{3}$ College of Agriculture and Human Sciences, Prairie View A\&M University, P.O. Box 519, MS 2008 Prairie View, TX 77446, USA. E-mail: ram.ray36@gmail.com

${ }^{4}$ Department of Physical Geography and Geoinformation Systems, University of Debrecen, 4032 Debrecen, Egyetem tér 1. Hungary, E-mail: szaboszilard.geo@gmail.com
} 
are usually calculated by statistical analysis using in-situ datasets (Prevot, L. et al. 1993; Magagi, R. and Kerr, Y. 1997; Frison, P. et al. 1998). KumAR, K. et al. (2012) used a genetic algorithm (GA) to calculate vegetation coefficients (A and $\mathrm{B}$ ) at the local scale using an empirical relationship between surface scattering and vegetation/crop biophysical parameters (using ENVISAT ASAR VV-pol data). Since these in-situ datasets are generally collected from specific observation sites, hence it is important to understand the usefulness of these observations in other regions. Consequently, in most of the crop models, crop coefficients vary from one location to another. It is important to develop a new approach or use existing approaches for the identification of crop coefficients, which does not require the in-situ observation of the biological and physical parameters of crops. Currently, WCM requires $\mathrm{V}_{1}$ and $\mathrm{V}_{2}$ vegetation parameters, which are associated with A, B, C, and D coefficients. Therefore, $V_{1}$ and $V_{2}$ must be precise, and easily available otherwise spatial variability of A, B, C, and D will be high. In the past, these two $\left(\mathrm{V}_{1}\right.$ and $\left.\mathrm{V}_{2}\right)$ vegetation parameters were estimated using extensive fieldwork within the study area; therefore, parameterization of WCM was easy at a local scale. RAwAT, K.S. et al. $(2017,2018)$ successfully estimated soil moisture using modified WCM (MWCM) by replacing $\mathrm{V}_{1}$ and $\mathrm{V}_{2}$ with NDVI value.
The study objective was to parameterize the MWCM using different combinations of vegetation indices. The combinations are categorized into Cases (I-IV) of combinations of vegetation indices such as Case $\mathrm{I}\left(\mathrm{V}_{1}-\mathrm{V}_{2}=\mathrm{NDVI}-\mathrm{EVI}\right)$, Case II $\left(\mathrm{V}_{1}-\mathrm{V}_{2}=\right.$ NDVI-NDVI), Case III $\left(\mathrm{V}_{1}-\mathrm{V}_{2}=\right.$ EVI$\mathrm{EVI})$ and Case IV $\left(\mathrm{V}_{1}-\mathrm{V}_{2}=\mathrm{EVI}-\mathrm{NDVI}\right)$; where NDVI is normalized vegetation index, and EVI is the enhanced vegetation index).

\section{Materials}

\section{Study area and ground data}

The Bathinda district study located in the state of Punjab India and is a region with wheat being the dominant crop (from $30^{\circ} 4^{\prime} 30^{\prime \prime} \mathrm{N}$ to $30^{\circ} 21^{\prime} 20^{\prime \prime} \mathrm{N}$ latitude and from $74^{\circ} 47^{\prime} 50^{\prime \prime}$ E to $75^{\circ} 10^{\prime} 00^{\prime \prime}$ E longitude) with average elevation of $210 \mathrm{~m}$ from sea level (Figure 1). The district of Bathinda lies in the extreme southwestern part of Punjab and far away from the Shivalik ranges in the North of the state. The normal annual rainfall of this region is about $408 \mathrm{~mm}, 80$ per cent of which is received during the southwestern monsoon season (First week of July to midSeptember) and remaining during the winter season. Dust storms are a regular feature in summer season when the temperature reaches to $47.0^{\circ} \mathrm{C}$ in the peak summer in May-

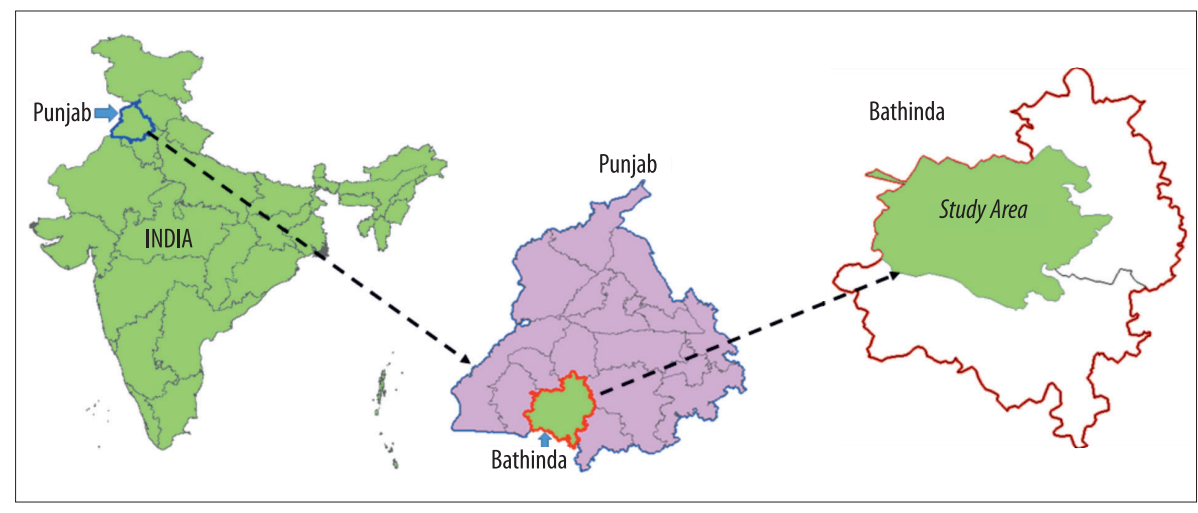

Fig. 1. Location map of the study area 
June, however, in winter during December and January, the minimum temperature at night could reach $0.0^{\circ} \mathrm{C}$.

The soil in the area is mostly loamy sand and sandy loam and contained $68-70$ per cent sand, 12-15 per cent silt and 18-21 per cent clay. Due to light texture, the water holding capacity of soil in the district varies from 2530 per cent depends on the clay content and organic matter present in the soil. The arid brown soils are calcareous in nature; these soils are imperfectly to moderately drained and siezoram soils the accumulation of calcium carbonate (CGWB, 2017). YADAV, B.K. et al. (2018) determined that the soils were low in available nitrogen $(\mathrm{N})$ low to medium in available phosphorous $(\mathrm{P})$ and medium to high in available potassium (K) content. They also found that there was wide variation in soil fertility status has developed on various landforms in Bathinda District, but the soils were low in available $\mathrm{N}$, low to medium in available $\mathrm{P}$ and medium to high in available K content. The measurements of soil moisture and vegetation parameters were carried out during the Sentinel-1 overpasses (dates are given in Table 1).

The Sentinel-1 mission provides active microwave data of C-band with $10 \mathrm{~m}$ resolution and has potential for soil moisture mapping. Further, European Space Agency (ESA) constellation of one more identical Sentinel-1A satellite on 25 April 2016 named Sentinel1B which has two microwave Synthetic Aperture Radar (SAR) sensors for improvement in temporal resolution.

Total of thirty imageries were acquired during the winter wheat crop growing period (details are provided in Table 1).
During each overpass of the satellite, insitu soil moisture measurements were performed using a time-domain reflectometer instrument (TDR, Field Scout ${ }^{\mathrm{TM}}$ TDR 300, Spectrum Technologies, Aurora, IL, United States). From sampling sites, soil moisture was measured using a TDR at a soil depth of $0-5 \mathrm{~cm}$. Calibration of the TDR instrument was performed as suggested by RAwAT, K.S. et al. $(2017,2018)$. The ancillary data, namely surface roughness, leaf area index (LAI), crop height, crop coverage and crop physiological states data were also collected.

\section{Landsat-8 and Sentinel-2 data}

A total of sixteen Sentinel-2 and Landsat- 8 datasets have been downloaded to estimate vegetation greenness in 2018 (Table 1). The spatial resolutions of Landsat- 8 and Sentinel-2 were $30 \mathrm{~m}$ and $10 \mathrm{~m}$, respectively. After pre-processing, vegetation greenness was calculated using the Normalized Difference Vegetation Index (NDVI) and Enhanced Vegetation Index (EVI).

Since the date of data acquisition difference was very small, hence a limited or no change was observed in NDVI and EVI value. Therefore, we did not performed any interpolation of NDVI and EVI value along with SAR images.

\section{Sentinel-1 data}

The Sentinel-1 operates at $5.4 \mathrm{GHz}$ frequency, and has four imaging modes, namely Stripmap model, Interferometric wide swath, extra-wide swath, and wave mode. In pre-

Table 1. Satellite data with a different date

\begin{tabular}{c|l|c}
\hline \multicolumn{1}{c|}{ Sensor } & \multicolumn{1}{|c}{ Dates in 2018} & $\begin{array}{c}\text { Spatial } \\
\text { resolution }\end{array}$ \\
\hline Sentinel-1A/B & January (20, 24), February $(1,5,13,17,25)$, March $(1,9,13,21,25)$, April $(6,14)$ & $10 \mathrm{~m}$ \\
Sentinel-2A/B & January (1, 27), February $(1,6,12,18)$, March $(1,6)$, April $(7,15)$ & $10 \mathrm{~m}$ \\
Landsat-8 & January (8, 17), February $(14,22,26)$, March (30) & $30 \mathrm{~m}$ \\
\hline
\end{tabular}


sent research work, S1 TOPS-model SLC data of an interferometric wide swath mode has been used for soil moisture estimation.

The pre-processing (radiometric and orthorectification) was performed according to the Sentinel-1 user data handbook. The Sentinel-1 data sets were processed in the SNAP platform (http://step.esa.int/main/toolboxes/ snap). The Sentinel-1 imageries were acquired in VV and VH polarization with an incidence angle near to $38^{\circ}$. We processed only $\mathrm{VV}$ polarization in our study because past studies (RAwAT, K.S. et al. 2019a, b) showed that $\mathrm{VH}$ polarization does not provide relevant crop/soil information with WCM for our study area.

Time Domain Reflectometer (TDR) instrument

The instrument TDR with a $7.5 \mathrm{~cm}$ probe length was used to collect in-situ soil moisture. TDR has wide spectrum frequency; it also works in C-band frequency as the Sentinel-1. TDR is a lightweight, portable instrument, it was used for in-situ observation. Besides, TDR may be used to get a large number of measurements over a short period of time (within satellite overpass of the study area) (RAwAT, K.S. et al. 2017, 2018, 2019a, b).

\section{Methods}

The Sentinel-1 data sets were processed for only VV polarization and generated backscattering coefficient $\left(\sigma^{\circ}\right)$ which is known as total $\sigma^{0}$ (or $\sigma_{\text {total }}^{0}$ ) because two $\sigma^{0}$ contribute in $\sigma_{\text {total }}^{0}$ backscattering from soil $\sigma^{0}\left(\sigma_{\text {soil }}^{0}\right)$ and backscattering from vegetation $\left(\sigma^{0}{ }_{\text {veg }}\right)$. The $\sigma_{\text {total }}^{0}$ of in-situ observation sites were derived using SNAP software. Similarly, NDVI and EVI value of each in-situ measurement sites were derived from the Sentinel- 2 and Landsat- 8 data. There was no need for re-sampling of Landsat- 8 into the Sentinel- 2 or Sentinel-2 into Landsat- 8 because our study area was homogenous and the size of the in-situ measurement plots was of dimensions more than $30 \mathrm{~m} \times 30 \mathrm{~m}$.
The MWCM was used to develop a semiempirical model for soil moisture estimation using microwave data. In MWCM, vegetation descriptors (or $V_{1}$ and $V_{2}$ ) were replaced by a couple of vegetation indices as Cases (I-IV) (as $\mathrm{V}_{1}-\mathrm{V}_{2}=$ NDVI-EVI, $\mathrm{V}_{1}-\mathrm{V}_{2}=$ NDVI-NDVI, $\mathrm{V}_{1}-\mathrm{V}_{2}=$ EVI-EVI; $\mathrm{V}_{1}-\mathrm{V}_{2}=$ EVI-NDVI; where NDVI $=$ normalized vegetation index [BALA, A. et al. 2015; RAwAT, K.S. et al. 2017, 2019a, b] and $\mathrm{EVI}=$ enhances vegetation index).

\section{Modified Water Cloud Model (MWCM)}

The WCM has a great possibility to diminish the effect of vegetation by computing the $\sigma^{0}{ }_{\text {veg }}$. It can be expressed by the following equation (1):

$$
\sigma_{\text {total }}^{0}(d B)=\sigma_{\text {veg }}^{0}+\sigma_{\text {veg+soil }}^{0}+\tau^{2}{\sigma^{0}}_{\text {soil }} \text {, eq. } 1
$$

For a given radar signal, $\sigma^{0}$ from the bare soil has a linear function of the soil moisture with depth $(0.0-7.5 \mathrm{~cm}$ ) (АттемA, E. and UlABY, F.T. 1978) and $\sigma^{0}{ }_{\text {veg+soil }} \approx 0$; therefore, eq. 1 can be modified as:

$$
\begin{array}{ccc}
\sigma_{\text {total }}^{0}(d B)=\sigma_{\text {veg }}^{0}+\tau^{2} \sigma_{\text {soil }}^{0}, & \text { eq. } 2 \\
\text { where: } & \sigma^{0}{ }_{\text {veg }}(d B)=A V_{1} \cos \left(1 \tau^{2}\right), & \text { eq. } 3 \\
\tau^{2}=\exp \left(2 B V_{2} / \cos \theta\right), & \text { eq. } 4 \\
\sigma_{\text {soil }}^{0}(d B)=C+D \cdot S M, & \text { eq. } 5
\end{array}
$$

where, $\theta$ is incident angle; $A$ and $B$ are vegetation coefficients that depend on the type of canopy, while coefficients $C$ and $D$ are soil dependent, $S M$ is soil moisture. $\mathrm{V}_{1}$ and $\mathrm{V}_{2}$ are canopy parameters and WCM was modified by changing these parameters $\left(\mathrm{V}_{1}\right.$ and $\left.\mathrm{V}_{2}\right)$ by NDVI or EVI. Magagi, R. and KerR, Y. (1997) investigated that due to change in vegetation states, canopy properties (bio and physical) change temporally. Therefore, a vegetation index is capable of explaining vegetation growth states.

\section{Model parametrization}

Based on Magagi, R. and KerR, Y. (1997), we have replaced $V_{1}$ and $V_{2}$ by a pair of vegetation 
indices (by applying in eq. 3 and 4), namely NDVI and EVI, since no previous work was found on replacing particular vegetation parameter (e.g. $V_{1}$ or $V_{2}$ ) by specific vegetation index (e.g. NDVI or EVI) to obtain optimal value of MWCM coefficients. However, NDVI and EVI can describe the winter wheat crop canopy as canopy descriptors. We tested different possible pairs of NDVI and EVI for the parametrization of the MWCM. We assumed that different possible combinations of NDVI and EVI would be better to incorporate the spatial water content/status, its spatial distribution within a confined volume, and would stand out an accurate simulation of total $\sigma^{0}$. The inverse distance method was applied for interpolation to generate the spatial maps of soil moisture (Mishra, A. et al. 2009).

\section{Vegetation index (NDVI/EVI)}

Crop mapping and environmental research commonly use NDVI (GARRoutTE, E. et al. 2016). Huete, A.R. (1988) investigated that NDVI responses were high for canopy background variations and showed saturated signals for high biomass conditions. EVI, suggested by QI, J. et al. (1994), improves sensitivity over dense vegetation conditions without the effect of the canopy background by minimizing canopy-soil variations (Huete, A.R. et al. 2002). We had selected two vegetation indices, and these vegetation indices were developed using equations (eq. 6, 7, 8 and 9). The particular band (blue, red, and near-infrared bands of Landsat-8 and Sentinel-2A/B) after atmospheric correction and conversion of digital number into reflectance of particular bands.

NDVI and EVI were used for the parametrization of vegetation and soil coefficients of MWCM (eq. 6 and eq. 7 for Landsat 8; eq. 8 and eq. 9 for Sentinel-2):

$$
\begin{gathered}
E V I=2.5 \cdot \frac{(\text { Band5 }- \text { Band } 4)}{(\text { Band5 }+6 \cdot \text { Band } 4-7.5 \cdot \text { Band } 2+1)} \text { eq. } 6 \\
N D V I=\frac{(\text { Band5 }- \text { Band } 4)}{(\text { Band } 5+\text { Band } 4)} \quad \text { eq. } 7
\end{gathered}
$$

$$
\begin{gathered}
\text { NDVI }=\frac{(\text { Band } 8-\text { Band } 4)}{(\text { Band } 8+\text { Band } 4)} \quad \text { eq. } 8 \\
E V I=2.5 \cdot \frac{(\text { Band } 8-\text { Band } 4)}{(\text { Band } 8+2.4 \cdot \text { Band } 4+1)} \text { eq. } 9
\end{gathered}
$$

where, the value of 2.5 in eq. 6 and 9 is a gain factor while 7.5 and 2.4 in eq. 6 and 9 (https://webapps.itc.utwente.nl/librarywww/ papers_2017/msc/nrm/adan.pdf) are coefficients, used to reduce aerosol effects and value 1 is the soil adjustment factor.

\section{Model coefficients ( $A, B, C$, and $D$ ) estimation}

Images of the study period were downloaded of 20/01/2018 to14/04/2018. This study assumed that the roughness over the crop was constant during the study period because of the single wheat crop, a slight change in roughness. Hence the number of unknown variables reduces into four for MWCM: coefficients of A, B, C, and $\mathrm{D}$ estimation errors (for coefficients) due to different factors (e.g. SAR sensor measurement error, optical sensor measurement error and NDVI estimation error) were also reduced. This process diminishes the effects over coefficients. The following two steps were used to prepare data for the optimal value of coefficients:

- Measured $\sigma^{0}$ at soil moisture sampling (using TDR) point from each corresponding to the Sentinel-1 data.

- The NDVI was calculated at each sampling point in the series of data.

The iterative optimization method was applied in SigmaPlot-12.0 to estimate the model coefficients (A, B, C and D). These model coefficients are important in predicting in $\sigma^{0}$ using the MWCM.

\section{Evaluation of observed and estimated $\sigma^{0}$}

In this study, the estimated VV polarized $\sigma^{0}$ with the help of possible combinations of NDVI and EVI as canopy descriptor and generated MWCM coefficients were tested with observed $\mathrm{VV \sigma}^{0}$ of Sentinel-1 using statistical 
tests and method as explained in RAwAT, K.S. et al. $(2017,2018)$. Statistical tests are the way to evaluate the accuracy of predicted data compared to observed data. However, there must be a sufficient number of datasets to draw a conclusion using statistical tests.

\section{Results and discussion}

For the simulation of $\mathrm{VV} \sigma^{0}$, the vegetation and soil coefficients were estimated for four combinations of NDVI and EVI for $\mathrm{V}_{1}$ and $\mathrm{V}_{2}$ (Table 2). The $\mathrm{C}$ and $\mathrm{D}$ soil coefficients have almost fixed values for each of the four combinations of vegetation indices (VIs) for $\mathrm{VV} \sigma^{0}$; therefore, $\mathrm{C}$ and $\mathrm{D}$ are free from canopy properties in the MWCM. Also, this type of interpretation can only be driven when $V_{1}$ and $V_{2}$ replaced by different vegetation indices combinations. If $\mathrm{V}_{1}$ and $\mathrm{V}_{2}$ are replaced by same VI (may be NDVI or EVI) then it cannot be concluded that $\mathrm{C}$ and $\mathrm{D}$ are independent of canopy because same NDVI $\left(\mathrm{V}_{1}\right)$-NDVI $\left(\mathrm{V}_{2}\right)$ or EVI $\left(\mathrm{V}_{1}\right)$-EVI $\left(\mathrm{V}_{2}\right)$ combinations of VI gives us only one value of $C$ and $D$. The value of $\mathrm{A}$ and $\mathrm{B}$ are different from different possible combinations of VIs (see Table 2). The values of A and B are completely governed by canopy properties (an orientation of leaf, the water content in leaf, and chlorophyll). It is further clarification of why more than one VI should be used for parameterization of MWCM. Table 2, non-zero values of A and B indicate that we cannot ignore the contribution of a canopy in microwave analysis.

MWCM parameterization using VIs was conducted by minimizing the RMSE with the best $\mathrm{R}^{2}$ value between observed and predicted VV $\sigma^{0}$ to optimize the effective unknown coefficients (A, B, C, and D). The comparative results of RMSE and $R^{2}$ between observed and predicted VV $\sigma^{0}$ from different vegetation parameters in combination are presented in Table 2 and shown in Figure 2.

MWCM Parameterization I: Estimation of $A, B$, and $C, D$ coefficients using $N D V I\left(V_{1}\right)-N D V I\left(V_{2}\right)$

NDVI is a commonly used index to monitor crop canopy, health and spatial distribution of vegetation during the growing season in agriculture. Therefore, NDVI-NDVI combination (case I) was used instead of $\mathrm{V}_{1}-\mathrm{V}_{2}$ vegetation parameters in MWCM. The graphical simulation of generated $\mathrm{VV} \sigma^{0}$ to $\mathrm{VV} \sigma^{0}$ from microwave data over the wheat crop (Figure 2, a). A total of 82 and observed data (NDVI and soil moisture) points (as input for $\sigma^{0}$ simulation) were used for NDVI-NDVI performance for predicting $\mathrm{VV} \sigma^{0}$ from MWCM. The model simulated VV $\sigma^{0}$ with a good $\mathrm{R}^{2}$ value of 0.61 , while the RMSE was highest in this combination (see Table 2). The NDVI represents the crop canopy solely in terms of its biophysical properties, and canopy background incorporates the dielectric properties.

MWCM Parameterization II: Estimation of $A, B$, and $C, D$ coefficients using $\operatorname{NDVI}\left(V_{1}\right)-E V I\left(V_{2}\right)$

NDVI and EVI combination (case II) was used as the canopy descriptors in MWCM. The $\mathrm{R}^{2}$

Table 2. Vegetation and soil coefficients with different possible combinations of vegetation parameters

\begin{tabular}{c|l|c|c|c|c|c|c}
\hline \multirow{2}{*}{\begin{tabular}{c} 
Canopy parameters \\
\multicolumn{2}{c|}{ of MWCM }
\end{tabular}} & \multicolumn{5}{|c|}{ MWCM coefficients } & \multirow{2}{*}{ RMSE } & \multirow{2}{*}{$\mathrm{R}^{2}$} \\
\cline { 2 - 6 } \multicolumn{1}{c|}{$\mathrm{V}_{1}$} & $\mathrm{~V}_{2}$ & $\mathrm{~A}$ & $\mathrm{~B}$ & $\mathrm{C}$ & $\mathrm{D}$ & \\
\hline NDVI & NDVI & 3.99 & 8.38 & 11.33 & 0.03 & 0.89 & 0.61 \\
NDVI & EVI & -2.89 & 0.418 & 11.21 & 0.02 & 0.73 & 0.68 \\
EVI & NDVI & -1.25 & 0.018 & 11.32 & 0.02 & 0.87 & 0.59 \\
EVI & EVI & -5.65 & 0.171 & 11.30 & 0.15 & 0.79 & 0.63 \\
\hline
\end{tabular}



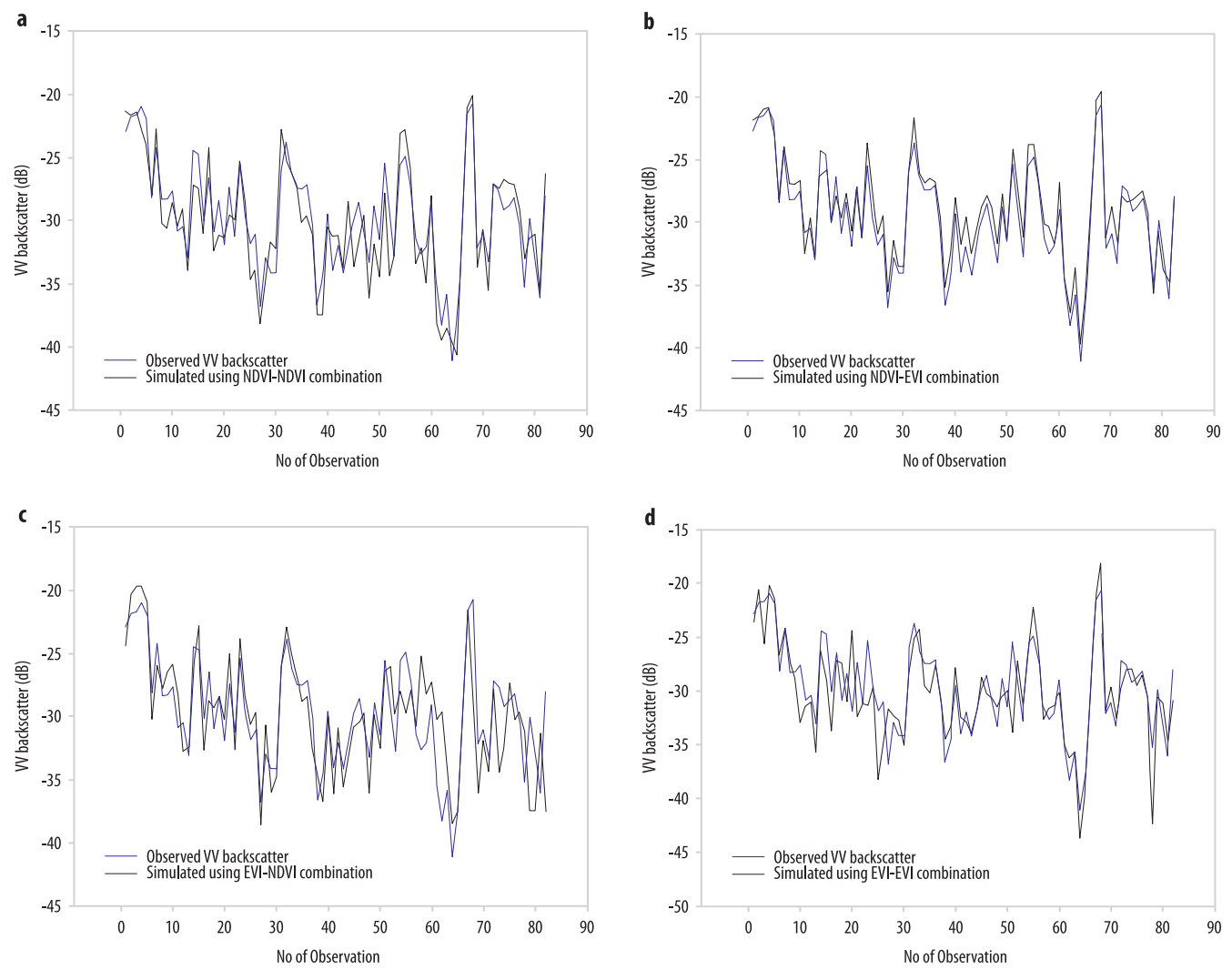

Fig. 2. Observed v/s simulated VV backscatter from the case I-IV. MWCM Parameterization I-IV: Estimation of $\mathrm{A}, \mathrm{B}$, and $\mathrm{C}$, D coefficients using $\mathbf{a}=\operatorname{NDVI}\left(\mathrm{V}_{1}\right)$-NDVI $\left(\mathrm{V}_{2}\right) ; \mathbf{b}=\operatorname{NDVI}\left(\mathrm{V}_{1}\right)$-EVI $\left(\mathrm{V}_{2}\right) ; \mathbf{c}=\operatorname{EVI}\left(\mathrm{V}_{1}\right)$-NDVI $\left(\mathrm{V}_{2}\right)$; $\mathbf{d}=\operatorname{EVI}\left(\mathrm{V}_{1}\right)$-EVI $\left(\mathrm{V}_{2}\right)$.

and RMSE (0.68 and 0.73, respectively, see Table 2) show good correlation (Figure 2, b). The MWCM retrieved VV $\sigma^{0}$ with a good accuracy based on statistical tests. It may be due to both conditions of crop/vegetation canopy information with a background (NDVI) and canopy information without the background (EVI). This possible combination also indicates that $\mathrm{V}_{1}$ slightly dependent on canopy background while $\mathrm{V}_{2}$ is independent of canopy background information (because EVI is free from canopy background information (QI, J. et al. 1994; Huete, A.R. et al. 2002). Therefore, both VIs combinations make a good prediction of $\mathrm{VV} \sigma^{0}$ from MWCM and moderately improves the performance.
MWCM Parameterization III: Estimation of $A, B$, and $C, D$ coefficients using EVI $\left(V_{1}\right)-N D V I\left(V_{2}\right)$

The case III of VIs was EVI $\left(\mathrm{V}_{1}\right)$-NDVI $\left(\mathrm{V}_{2}\right)$. A significant decrement was witnessed when the combination of EVI and NDVI was observed. The MWCM shows below the average $R^{2}(0.62)$ value in four possible combinations of two VIs (see Table 2). Based on statistical tests, MWCM works with low efficiency because this combination has a low $\mathrm{R}^{2}$ value of 0.59 (see Table 2; Figure 2, c). The third combination of VIs was just opposite of case II, and from this condition, we have also concluded that $\mathrm{V}_{1}$ slightly supports the canopy background in MWCM. 
MWCM Parameterization IV: Estimation of $A$, $B$, and $C, D$ coefficients using $E V I\left(V_{1}\right)-E V I\left(V_{2}\right)$

The case IV was EVI $\left(\mathrm{V}_{1}\right)$-EVI $\left(\mathrm{V}_{2}\right)$. This combination gives a marginal improvement in the performance of MCM with the $\mathrm{R}^{2}$ of 0.63 for VV $\sigma^{0}$ (see Table 2). There was a second highest preferable value of RMSE and $\mathrm{R}^{2}$ for $\mathrm{VV} \sigma^{0}$ in comparison to MWCM with different combinations of VIs (Figure 2, d). Therefore, we can infer that EVI depicts the wheat crop canopy in a better way for the $V_{2}$ parameter in the MWCM.

\section{Evaluation of observed and estimated $\sigma^{0}$}

In this study, statistical tests showed that NDVI-EVI combination had the potential to provide good results with the agreement of nine statistical tests. The combination of NDVI-EVI as a combination of vegetation parameters or canopy descriptor $\left(\mathrm{V}_{1}-\mathrm{V}_{2}\right)$ showed the least RMSE of $0.81 \mathrm{~dB}$ between observed and predicted VV $\sigma^{0}$ while highest $\mathrm{R}^{2}$ value of 0.98 among other VIs combination for observed and predicted VV $\sigma^{0}$ (Table 3 ).

The accuracy of retrieval of $\mathrm{VV}^{0}$ could be different when different combinations of VIs were chosen. WANG, L. et al. (2019) also found the accuracy of retrieval depends on the selection of VIs. The accuracy of retrieval of $\mathrm{VV \sigma}^{0}$ was high when the $\mathrm{V}_{1}$ replaced by NDVI and $V_{2}$ by EVI (see Table 3). Also, when the $\mathrm{V}_{1}$ replaced by EVI and the $\mathrm{V}_{2}$ by NDVI, retrieval accuracy decreases, which means that canopy background influences the $\mathrm{V}_{1}$ parameter in MWCM while $\mathrm{V}_{2}$ may be canopy background free. Because EVI is canopy background free while NDVI showed canopy as well as litter bit canopy background (soil) information, this analysis also revealed that any combination of VIs does not have much effect on soil coefficients $C$ and $D$, because $\mathrm{C}$ and D depend entirely on soil properties (e.g., bulk density, soil texture, etc.) rather than vegetation properties. The $C$ and $D$ parameters were fixed by using linear equation eq. 5. Therefore, for any combination of VIs, the value of $C$ and $D$ does not change much. It was found that the errors in vegetation or canopy descriptors were sensitive to errors in the retrieval of $\mathrm{VV} \sigma^{0}$ ( $\mathrm{LIU}, \mathrm{C}$. and SHI, J. 2016).

\section{Conclusion}

In this study, WCM was modified using vegetation/canopy descriptor to simulate $V V \sigma^{0}$. The current research focused on the parameterization of MWCM. In the current research work, a combination of vegetation indices and backscattering (VV) simulated

Table 3. Statistical evaluation of estimated with respect observed VV backscatter $\left(\sigma^{0}\right)$, based on NDVI as crop canopy descriptor and on investigated coefficients

\begin{tabular}{l|c|c|c|c|c|c|c}
\hline Observed & -27.24 & -31.39 & -25.24 & -29.59 & -31.88 & -30.98 & -36.88 \\
$\mathrm{E}_{\text {NDVI-NDVI }}$ & -29.28 & -29.93 & -24.11 & -27.49 & -35.16 & -33.98 & -39.45 \\
$\mathrm{E}_{\text {NDVI-EVI }}$ & -26.86 & -30.95 & -25.21 & -29.2 & -31.91 & -31.03 & -38.89 \\
$\mathrm{E}_{\text {EVI-NDVI }}$ & -28.28 & -30.93 & -25.11 & -28.49 & -34.73 & -32.98 & -37.45 \\
$\mathrm{E}_{\text {EVI-EVI }}$ & -25.28 & -25.99 & -24.70 & -27.96 & -33.66 & -31.09 & -36.22 \\
\hline \multicolumn{2}{l}{ Statistical performance measures } & NDVI-NDVI & NDVI-EVI & EVI-NDVI & EVI-EVI \\
\hline$R^{2}$ & 0.83 & 0.98 & 0.90 & 0.75 \\
RMSE & 2.34 & 0.81 & 1.46 & 2.38 \\
R-RMSE & & 0.08 & 0.02 & 0.05 & 0.08 \\
MAE & & 0.89 & 0.12 & 0.68 & 1.19 \\
NRMSE & & -0.08 & -0.03 & -0.05 & -0.08 \\
MAE & & 0.89 & 0.12 & 0.68 & -1.19 \\
SEE & & -1.10 & 0.87 & 1.58 & 2.57 \\
RMSE, \% & & 1.03 & -0.38 & -0.69 & -1.12 \\
IR & & & 1.00 & 1.02 & 0.96 \\
\hline
\end{tabular}


from Sentinel-1 was studied. The assumed hypothesis was that surface roughness during wheat crop period was constant. A total of four combinations were tested for the comparison of $\mathrm{VV \sigma}^{0}$ to the observed $\mathrm{VV} \sigma^{0}$. Our results revealed that MWCM could be parameterized with NDVI and EVI as canopy descriptors. The basis of optimization of A, B, C and D by reducing RMSE between MWCM predicted and Sentinel-1 observed $\mathrm{VV} \sigma^{0}$. The retrieval of $\mathrm{VV} \sigma^{0}$ converges to the correct (with good accuracy or free from errors) values of the vegetation or canopy descriptors.

Acknowledgement: Authors KSR and SKS express thanks to Ministry of Earth Sciences (MoES), Government of India, India for providing the financial support (MoES/16/13/2016-RDEAS) to carry out this research work. The author SS was supported by the Higher Education Institutional Excellence Programme (NKFIH-1150-6/2019) of the Ministry of Innovation and Technology in Hungary, within the framework of the $4^{\text {th }}$ thematic programme of the University of Debrecen.

\section{REFERENCES}

Attema, E. and Ulaby, F.T. 1978. Vegetation modelled as a water cloud. Radio Science 13. (1): 357-364.

Bala, A., Pawar, S.P., Misra, A.K. and Rawat, K.S. 2015. Estimation and validation of actual evapotranspiration for wheat crop using SEBAL model over Hisar District (Haryana), India. Current Science 113. (1): 134-141.

Beck, H.E., Jeu, R.A.M., Schellekens, J., DiJK, A.I.J.M. and BRUIJNZEEL, L.A. 2009. Improving curve number based storm runoff estimates using soil moisture proxies. IEEE Journal of Selected Topics in Applied Earth Observations and Remote Sensing 2. (4): 250-259.

Berthet, L., Andréassian, V., Perrin, C. and Javelle, P. 2009. How crucial is it to account for the antecedent moisture conditions in flood forecasting? Comparison of event-based and continuous approaches on 178 catchments. Hydrology Earth System Science 13. (6): 819-831.

Bindlish, R., Jackson, J.T., Gasiewski, A.J., KLein, M. and NJoKU, E.G. 2006. Soil moisture mapping and AMSR-E validation using the PSR in SMEX02. Remote Sensing of Environment 103. (2): 127-139.

CGWB 2017. Ground Water Information Bathinda District, Punjab. Chandigarh, North Western Region. Central Ground Water Board, Ministry of Water
Resources Government of India. Available at http:// cgwb.gov.in/District_Profile/Punjab/Bathinda.pdf

Dezső, J., Czigány, S., Nagy, G., Pirkhoffer, E., SŁowiK, M. and Lóczy, D. 2019. Monitoring soil moisture dynamics in multilayered Fluvisols. Bulletin of Geography. Physical Geography Series 16. (1): 131-146.

Frison, P., Mougin, E. and Hiernaux, P. 1998 Observations and interpretation of seasonal ERS-1 wind scatterometer data over northern Sahel (Mali). Remote Sensing of Environment 63. 233-242.

Garroutte, E., Hansen, A. and Lawrence, R. 2016. Using NDVI and EVI to map spatiotemporal variation in the biomass and quality of forage for migratory elk in the Greater Yellowstone Ecosystem. Remote Sensing 8(5):404. Available at https://doi. org/10.3390/rs8050404.

Hegedús, P., Czigány, S., Balatonyi, L. and Pirkhoffer, E. 2013. Analysis of soil boundary conditions of flash floods in a small basin in SW Hungary. Open Geosciences 5. (1): 97-111.

Hegedús, P., CZigány, S., Pirkhoffer, E., Balatonyi, L. and HiскEY, R. 2015. Analysis of spatial variability of near-surface soil moisture to increase rainfallrunoff modelling accuracy in SW Hungary. Open Geosciences 7. (1): 126-139.

Hornberger, G.M. 1998. Elements of Physical Hydrology. Baltimore, MD, USA, Johns Hopkins University Press.

Huete, A.R, Didan, K., Miura, T., Rodriguez, E.P., Gao, X. and Ferreira, L.G. 2002. Overview of the radiometric and biophysical performance of the MODIS Vegetation indices. Remote Sensing of Environment 83. 195-213.

Huete, A.R. 1988. A soil-adjusted vegetation index (SAVI). Remote Sensing of Environment 25. (3): 295-309.

Kumar, K., Prasad, K.S.H. and Arora, M.K. 2012. Estimation of water cloud model vegetation parameters using a genetic algorithm. Hydrological Sciences Journal 57. 776-789.

Liv, C. and SHI, J. 2016. Estimation of vegetation parameters of Water Cloud Model for global soil moisture retrieval using Time-Series L-Band Aquarius Observations. IEEE Journal of Selected Topics in Applied Earth Observations and Remote Sensing 9. (12): 5621-5633.

Magagi, R. and KerR, Y. 1997. Retrieval of soil moisture and vegetation characteristics by use of ERS-1 wind scatterometer over arid and semi-arid areas. Journal of Hydrology 188. 361-384.

Mishra, A., Gairola, R.M., Varma, A.K., Sarkar, A. and Agarwal, V.K. 2009. Rainfall retrieval over Indian land and oceanic regions from SSM/I microwave data. Advances in Space Research 44. 815-823.

Paloscia, S., Pettinato, S., Santi, E., Notarnicola, C., Pasolli, L. and Reprucci, A. 2013. Soil moisture mapping using Sentinel-1 images: Algorithm 
and preliminary validation. Remote Sensing of Environment 134. 234-248.

Pasolli, L., Notarnicola, C., Bertoldi, G., Bruzzone, L., Remelgado, R., Greifeneder, F., Niedrist, G., Chiesa, S.D., Tappeiner, U. and Zebisch, M. 2015. Estimation of soil moisture in mountain areas using SVR technique applied to multiscale active radar images at C-band. IEEE Journal of Selected Topics in Applied Earth Observations and Remote Sensing 8. (1): 262-283.

Pathe, C., Wagner, W., Sabel, D., Doubkova, M. and BASARA, J.B. 2009. Using ENVISAT ASAR global mode data for surface soil moisture retrieval over Oklahoma, USA. IEEE Transactions on Geoscience and Remote Sensing 47. (2): 468-480.

Prevot, L., Dechambre, M., Taconet, O., VidalMadjar, D., Normand, M. and Gallej, S. 1993. Estimating the characteristics of vegetation canopies with airborne radar measurements. International Journal of Remote Sensing 14. 2803-2818.

Qi, J., Chehbouni, A., Huete, A.R., Kerr, Y.H. and Sorooshian, S. 1994. A modified soil adjusted vegetation index. Remote Sensing of Environment. 48. (2): 119-126.

Rawat, K.S., Sehgal, V.K., Pradhan, S. and Ray, S.S. 2017. Retrieval and validation of soil moisture from FRS-1 data set of Radar Imaging Satellite (RISAT-1). Arabian Journal of Geosciences 10. (445): 1-10. Doi: 10.1007/s12517-017-3195-6.

Rawat, K.S., SehgaL, V.K. and Ray, S.S. 2018. Semiempirical model for retrieval of soil moisture using RISAT-1 C-band data over a sub-tropical semi arid area of Rewari District State of Haryana (India). Journal of Earth System Science 127(2). Doi: 10.1007/ s12040-018-0919-2.
Rawat, K.S., Singh, S.K. and PaL, R.K. 2019a. Synergetic methodology for estimation of soil moisture over agricultural area using Landsat- 8 and Sentinel-1 satellite data. Remote Sensing Applications: Society and Environment 15(100250).

Rawat, K.S., Singh, S.K. and RAY, R.L. 2019b. An integrated approach to estimate surface soil moisture in agricultural lands. Geocarto International 2019(11). Available at https: //doi.org/10.1080/10106049.2019. 1678674.

Shi, J.C., WANG, J., Hsu, A.Y., Neill, P.E.O. and ENGMAN, E.T. 1997. Estimation of bare surface soil moisture and surface roughness parameter using L-band SAR image data. IEEE Transactions on Geoscience and Remote Sensing 35. (5): 1254-1266.

WANG, L., HE, B., BAI, X. and XING, M. 2019. Assessment of different vegetation parameters for parameterizing the coupled water cloud model and advanced integral equation model for soil moisture retrieval using time series Sentinel-1A data. Photogrammetric Engineering \& Remote Sensing 85. (1): 43-54.

YAdAV, B.K., Sidhu, A.S. and Kumar, D. 2018. Distribution and indexation of plant available nutrients in Bathinda district of south-west Punjab, India. Journal of Soils and Crops 28. (1): 8-18. 